УДК 661.183 .2

\title{
Optimization of the Process of Synthesis of Porous Carbon Materials Obtained from the Bast Birch Bark by Means of Steam-Gas Activation
}

\author{
Ivan P. Ivanov*a, Nadezhda M. Mikova ${ }^{\text {a }}$, Robert Z. Pen \\ ${ }^{a}$ Institute of Chemistry and Chemical Technology SB RAS \\ 50 Akademgorodok, Krasnoyarsk, 660036, Russia \\ ${ }^{b}$ Siberian State Technological University, \\ 82 Mira, Krasnoyarsk, 660049, Russia
}

Received 12.10.2015, received in revised form 13.11.2015, accepted 07.12.2015

\begin{abstract}
The method of experimental and statistical analysis was used to study the influence of basic factors: carbonization temperature and duration of steam-gas one-stage process of carbonization-activation on the values of the parameters of porous structure of carbon adsorbents (CA) obtained from the bast birch bark. It was determined that the realization of process under optimal conditions allows to obtain from the birch bark the carbon sorbents, whose characteristics pore volume of $1.61 \mathrm{~cm} 3$ water $/ g$ and iodine adsorption of $75.2 \%$ correspond to the of industrial carbon sorbent BAU derived from birch wood.
\end{abstract}

Keywords: bast of birch bark, pore volume, physical activation, carbon sorbent, process optimization, mathematical model.

DOI: $10.17516 / 1998-2836-2015-8-4-521-532$.

(C) Siberian Federal University. All rights reserved

* Corresponding author E-mail address: ivanov@icct.ru 


\title{
Оптимизация процесса синтеза
}

пористых углеродных материалов,

получаемых из луба коры березы

методом парогазовой активации

\author{
И.П. Иванов ${ }^{a}$, Н.М. Микова ${ }^{a}$, Р.3. Пен ${ }^{6}$ \\ ${ }^{a}$ Институт химии и химической технологии СО РАН \\ 50 Академгородок, Красноярск, 660036, Россия \\ ${ }^{6}$ Сибирский государственный технологический университет \\ 82 пр. Мира, Красноярск, 660049, Россия
}

\begin{abstract}
Методом экспериментально-статистического анализа изучено влияние основных факторов: температуры и продолжительности одностадийного процесса парогазовой карбонизацииактивации в аппарате кипящего слоя на значения параметров пористой структуры углеродных сорбентов, получаемых из луба коры березы. Установлено, что проведение процесса в оптимальных условиях позволяет получать из луба коры березы углеродные сорбенты (с объемом пор по воде 1,61 $\mathrm{cm}^{3} / 2$ и адсорбиионной по йоду 75,2 \%), соответствующие по своим характеристикам промышленному углеродному сорбенту БАУ, получаемому из древесины березы.
\end{abstract}

Ключевые слова: луб коры березы, объем пор, физическая активация, углеродный сорбент, оптимизация процесса, математическая модель.

\section{Введение}

Древесная кора является одним из самых распространенных биоресурсов в мире. Кора составляет от 9 до 15 \% от объема стволовой древесины [1]. Она, как правило, образуется в виде отходов при заготовке и промышленной переработке древесины. По данным на 2010 г., в мире было заготовлено около 1542 млн м ${ }^{3}$ круглого леса, что генерировало около 200 млн м ${ }^{3}$ коры [2].

Традиционно кора либо остается в лесу после рубки деревьев, либо используется в качестве топлива в лесной промышленности. Большие и концентрированные количества коры находятся на складах лесоперерабатывающей промышленности, таких как целлюлозно-бумажные комбинаты и малотоннажные лесоперерабатывающие предприятия. В тех случаях, когда кора не используется в качестве технологического топлива на месте, утилизация коры становится серьезной проблемой.

Вместе с тем сжигание коры экономически не выгодно, так как она имеет относительно низкую теплотворную способность и значительное содержание воды.

Перечисленные проблемы стимулируют проведение исследований, направленных на решение актуальной задачи утилизации коры. В последние годы наблюдается возрождение интереса к комплексной переработке возобновляемой растительной биомассы и ее отходам в 
качестве источника для производства химических веществ, материалов и энергии, т.е. более эффективного использования биомассы [3-5].

Наиболее перспективным вариантом утилизации коры считается переработка с получением химических продуктов и функциональных материалов, в частности углеродных сорбентов [6-9].

Получение пористых углеродных сорбентов из древесной коры возможно с использованием методов химической и физической активации и позволяет заменить более ценное древесное сырье.

Перспективным направлением получения пористых углеродных сорбентов из коры может быть технология, основанная на совмещенном процессе пиролиза и активации в аппаратах кипящего слоя. В сравнении со слоевыми процессами условия работы установок псевдоожиженного слоя характеризуются более высокими коэффициентами тепло- и массообмена, широтой диапазона варьирования основных параметров процесса, а также легкой адаптируемостью к изменению свойств исходного сырья.

Целью математической оптимизации процесса получения углеродных сорбентов из луба коры березы методом пиролиза-активации в аппаратах кипящего слоя являлось определение влияния независимых факторов (температура и продолжительность процесса активации) на значения выходных параметров: степени обгара, объема пор по воде и сорбционную активность по йоду.

\section{Экспериментальная часть}

Настоящая методика оптимизации осуществлена с целью математического моделирования условий, обеспечивающих наилучшие условия проведения процесса активации луба коры березы для получения углеродных сорбентов, соответствующих по своим характеристикам промышленному углеродному сорбенту БАУ, получаемому из древесины березы с объемом пор по воде не менее $1,5 \mathrm{~cm}^{3} / \Gamma$ и адсорбционной активностью по йоду не менее $70 \%$.

Математическая обработка результатов выполнена средствами пакета прикладных программ StatgraphicsCenturionXVI, блок DOE (DesignofExperiment), процедура MultiFactorCategorical [10].

В качестве переменных условий процесса выбраны наиболее важные факторы, определенные на основании априорной информации и результатов предварительных экспериментов температура $\mathrm{X}_{1}$ и продолжительность $\mathrm{X}_{2}$ процесса. Условия экспериментов (значения независимых переменных) приведены в табл. 1, столбцы 1 и 2.

Результаты экспериментов характеризовали следующими выходными параметрами:

$\mathrm{У}_{1}$ - степень обгара, \%;

$\mathrm{y}_{2}$ - объем пор по воде, $\mathrm{cm}^{3} / \Gamma$;

$\mathrm{y}_{3}$ - адсорбционная активность по йоду, \%.

Полученные в эксперименте значения выходных параметров отражены в табл. 1, столбцы 3,5 и 7.

Сравнительную сорбционную активность по йоду полученных сорбентов из луба коры березы и БАУ-А определяли по стандартной методике [11]. 
Таблица 1. Влияние условий парогазовой активации луба коры березы на характеристики получаемых углеродных сорбентов

\begin{tabular}{|c|c|c|c|c|c|c|c|}
\hline \multirow{2}{*}{\multicolumn{2}{|c|}{ Условия опытов }} & \multicolumn{6}{|c|}{ Результаты экспериментов } \\
\hline & & \multicolumn{2}{|c|}{ степень обгара, $\mathrm{Y}_{1}, \%$} & \multicolumn{2}{|c|}{$\begin{array}{c}\text { объем пор по воде, } \\
\mathrm{Y}_{2}, \mathrm{~cm}^{3} / \Gamma\end{array}$} & \multicolumn{2}{|c|}{$\begin{array}{c}\text { адсорбционная } \\
\text { активность по йоду, } \mathrm{Y}_{3}, \%\end{array}$} \\
\hline $\mathrm{X}_{1},{ }^{\circ} \mathrm{C}$ & $\mathrm{X}_{2}$, мин & эксперимент & прогноз & эксперимент & прогноз & эксперимент & прогноз \\
\hline 1 & 2 & 3 & 4 & 5 & 6 & 7 & 8 \\
\hline 700 & 15 & 41,2 & 41,1 & 0,93 & 0,89 & 37,4 & 37,4 \\
\hline 750 & 15 & 44,1 & 44,6 & 1,25 & 1,30 & 58,7 & 59,6 \\
\hline 800 & 15 & 49,4 & 48,8 & 1,54 & 1,54 & 72,8 & 72,1 \\
\hline 850 & 15 & 53,3 & 53,7 & 1,68 & 1,60 & 74,3 & 74,9 \\
\hline 900 & 15 & 59,7 & 59,4 & 1,46 & 1,50 & 68,9 & 68,1 \\
\hline 800 & 10 & 42,1 & 41,8 & 1,42 & 1,44 & 68,4 & 67,6 \\
\hline 850 & 10 & 44,8 & 45,1 & 1,53 & 1,51 & 70,1 & 70,9 \\
\hline 800 & 20 & 57,3 & 57,0 & 1,33 & 1,35 & 70,6 & 69,8 \\
\hline 850 & 20 & 63,2 & 63,5 & 1,42 & 1,40 & 71,4 & 72,2 \\
\hline
\end{tabular}

\section{Результаты и обсуждение}

В табл. 1 приведены результаты полученных экспериментальных величин.

Результаты математической обработки для каждого выходного параметра в отдельности приведены ниже.

Анализ влияния условий парогазовой активациии

на степень обгара углеродных сорбентов из луба коры березы

В табл. 2 отражены результаты дисперсионного анализа для получения математических моделей (уравнений регрессии) и вычисления оптимальных условий, связывающих характер зависимостей исходных переменных $\mathrm{X}_{1}$ (температуры активации) и $\mathrm{X}_{2}$ (продолжительность активации) с выходным параметром $\mathrm{Y}_{1}$ (степень обгара).

Уравнение регрессии, аппроксимирующее зависимость $\mathrm{Y}_{1}$ от $\mathrm{X}_{1}$ и $\mathrm{X}_{2}$, имеет следующий вид:

$$
\begin{aligned}
& Y_{1}=126,1-0,2327 X_{1}-4,278 X_{2}+0,0001424 X_{1}^{2}+ \\
& +0,0064 X_{1} X_{2}+0,02259 X_{2}^{2} .
\end{aligned}
$$

Выполненный дисперсионный анализ показал, что наиболее существенный вклад в суммарную дисперсию степени обгара вносит фактор $\mathrm{X}_{1}$ (температура). Вклад влияния температуры характеризуется максимальной величиной критерия эффективности $F(528,15)$ и минимальным уровнем значимости $\mathrm{P}(0,0002)$. При этом коэффициент детерминации $\mathrm{R}^{2}$ составляет $99,4 \%$.

Близость «экспериментальных точек» выходного параметра степени обгара к прямой линии, соответствующей расчетным (прогнозируемым) значениям $\mathrm{Y}_{1}$, указывает на хорошие прогностические свойства модели (уравнение регрессии). Этот результат корреспондируется с величиной коэффициента детерминации $\mathrm{R}^{2}$ (квадрат выборочного коэффициента), близкого по смыслу к коэффициенту множественной корреляции между выходным параметром степени 
Таблица 2. Результаты дисперсионного анализа для степени обгара (Y1)

\begin{tabular}{|c|c|c|c|c|c|}
\hline $\begin{array}{c}\text { Источники } \\
\text { дисперсии }\end{array}$ & $\begin{array}{c}\text { Суммы } \\
\text { квадратов }\end{array}$ & $\begin{array}{c}\text { Степени } \\
\text { свободы }\end{array}$ & $\begin{array}{c}\text { Средние } \\
\text { квадраты }\end{array}$ & $\begin{array}{c}\text { Дисперсионные } \\
\text { отношения, F }\end{array}$ & $\begin{array}{c}\text { Уровни } \\
\text { значимости, Р }\end{array}$ \\
\hline $\mathrm{A}: \mathrm{X}_{1}$ & 227,197 & 1 & 227,197 & 528,15 & 0,0002 \\
\hline $\mathrm{B}: \mathrm{X}_{2}$ & 115,52 & 1 & 115,52 & 268,54 & 0,0005 \\
\hline $\mathrm{AA}$ & 1,89055 & 1 & 1,89055 & 4,39 & 0,1270 \\
\hline $\mathrm{AB}$ & 2,56 & 1 & 2,56 & 5,95 & 0,0925 \\
\hline $\mathrm{BB}$ & 0,504145 & 1 & 0,504145 & 1,17 & 0,3583 \\
\hline $\mathrm{R}^{2}, \%$ & \multicolumn{5}{|c|}{} \\
\hline
\end{tabular}

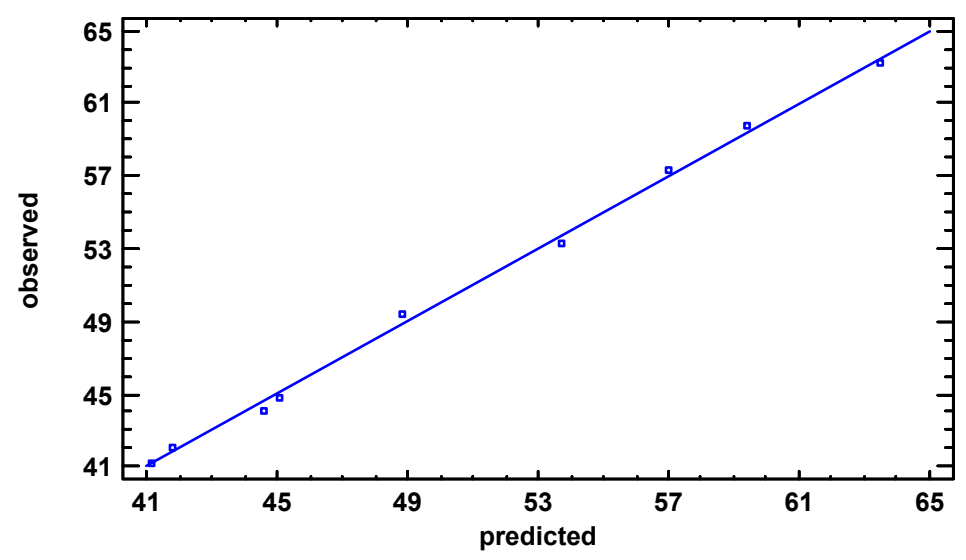

Рис. 1. Сравнение экспериментальных и расчетных значений $Y_{1}$ углеродных сорбентов из луба коры березы

обгара и переменными факторами. Относительно высокое значение коэффициента $\mathrm{R}^{2}=99,4 \%$ (коэффициент детерминации с поправкой на число степеней свободы) указывает на хорошее качество аппроксимации результатов наблюдений уравнением (1).

Прогностические свойства уравнения (1) видны из близости результатов опытов (столбец 3 в табл. 1) и прогнозируемыми моделью значениями (столбец 4). На это же указывает близкое к $100 \%$ значение коэффициента детерминации $R^{2}=99,4 \%$. Наглядное представление о качестве модели дает рис. 1. Близость точек к прямой подтверждает хорошее качество модели.

На рис. 2 приведена поверхность отклика, иллюстрирующая зависимость результата процесса (степени обгара) от переменных факторов $\mathrm{X}_{1}$ и $\mathrm{X}_{2}$. Поверхность отклика отражает факторные координаты, в которых значение степени обгара зависит от влияния переменных факторов.

Представленная на рис. 2 поверхность отклика, соответствующая уравнению (1) - зависимость $\mathrm{Y}_{1}$ от $\mathrm{X}_{1}$ и $\mathrm{X}_{2}$, показывает, что величина обгара почти равномерно увеличивается с ростом температуры и продолжительности процесса активации луба коры березы. На рис. 2 видны условия, при которых достигаются максимальная и минимальная величины обгара в пределах изученной области факторного пространства (диапазонов изменения температуры и 


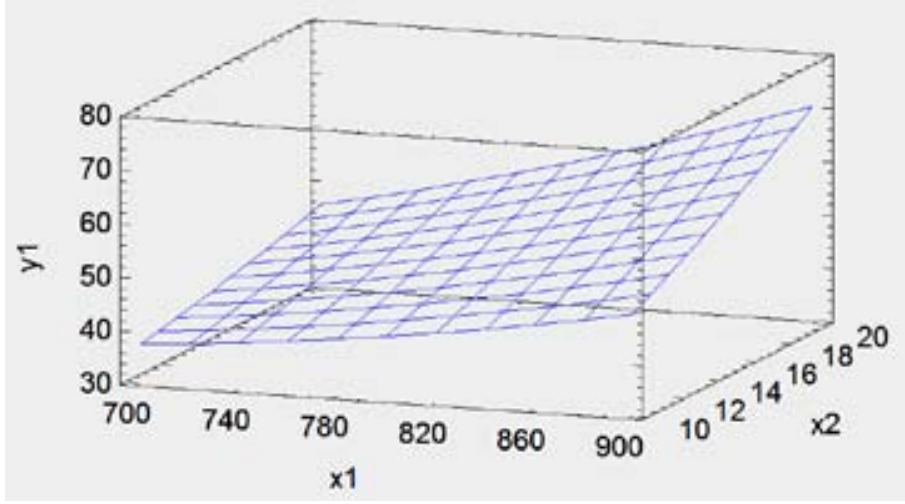

Рис. 2. Поверхность отклика зависимости степени обгара $\mathrm{Y}_{1}$ от условий процесса $\mathrm{X}_{1}$ и $\mathrm{X}_{2}$

продолжительности процесса): максимальное значение $\mathrm{Y}_{1}=70 \%$ соответствует температуре активации $900{ }^{\circ} \mathrm{Cи}$ продолжительности 20 мин, а минимальное значение $(37 \%)-$ при $700{ }^{\circ} \mathrm{C}$ в течение 10 мин.

Анализ влияния условий парогазовой активации

на объем пор углеродных сорбентов из луба коры березы

В табл. 3 приведены результаты дисперсионного анализа для получения математических моделей (уравнений регрессии) и вычисления оптимальных условий, связывающих характер зависимостей исходных переменных $\mathrm{X}_{1}$ (температуры активации) и $\mathrm{X}_{2}$ (продолжительность активации) с выходным параметром $\mathrm{Y}_{2}$ (объем пор).

Уравнение регрессии, аппроксимирующее зависимость $\mathrm{Y}_{2}$ от $\mathrm{X}_{1}$ и $\mathrm{X}_{2}$, имеет следующий вид:

$$
\begin{aligned}
& Y_{2}=-24,49+0,05836 X_{1}+0,2047 X_{2}-0,00003419 X_{1}^{2}- \\
& -0,0000400 X_{1} X_{2}-0,006056 X_{2}^{2} .
\end{aligned}
$$

Выполненный дисперсионный анализ показал, что наиболее существенный вклад в суммарную дисперсию объема пор вносит фактор $\mathrm{X}_{1}$ (температура). Вклад влияния температуры характеризуется максимальной величиной критерия эффективности $\mathrm{F}(61,89)$ и минимальным уровнем значимости $\mathrm{P}(0,0043)$. При этом коэффициент детерминации $\mathrm{R}^{2}$ составляет $91,1 \%$.

Близость «экспериментальных точек» выходного параметра объема пор к прямой линии, соответствующей расчетным (прогнозируемым) значениям объема пор, указывает на хорошие прогностические свойства модели (уравнение регрессии). Этот результат соотносится с величиной коэффициента детерминации $\mathrm{R}^{2}$ (квадрат выборочного коэффициента), близкого по смыслу к коэффициенту множественной корреляции между выходным параметром объема пор и переменными факторами и значением коэффициента $\mathrm{R}^{2}=91,1$ \% (коэффициент детерминации с поправкой на число степеней свободы), что указывает на хорошее качество аппроксимации результатов наблюдений уравнением (2).

Прогностические свойства уравнения (2) видны из близости результатов опытов (столбец 5 в табл. 1) и прогнозируемых моделью значений (столбец 6). На это же указывает 
достаточно высокое значение коэффициента детерминации $-R^{2}=91,1 \%$. Наглядное представление о качестве модели дает рис. 3. Близость точек к прямой подтверждает хорошее качество модели.

На рис. 4 приведены поверхности отклика, иллюстрирующие зависимость результата процесса (объема пор) от переменных факторов $\mathrm{X}_{1}$ и $\mathrm{X}_{2}$. Поверхность отклика отражает факторные координаты точки, в которой значение объема пор достигает оптимальной величины в зависимости от влияния переменных факторов.

На рис. 4 отражено, что зависимость объема пор от переменных факторов искривлена (нелинейная). При этом объем пор существенно зависит от температуры активации (в пределах изученного диапазона температуры), тогда как влияние продолжительности процесса активации проявилось в меньшей степени. Точка, соответствующая условиям, при которых объем пор максимален, находится внутри изученной области факторного пространства.

Из представленных на рис. 5 изолиний поверхности отклика, зависимости объема пор ( $\left.\mathrm{Y}_{2}\right)$ от условий процесса $\mathrm{X}_{1}$ и $\mathrm{X}_{2}$ видна точка, в которой $\mathrm{Y}_{2}$ достигает максимального значения, в центре эллипса. Координаты этой точки соответствуют температуре процесса активации

Таблица 3. Результаты дисперсионного анализ для объема пор $\left(\mathrm{Y}_{2}\right)$

\begin{tabular}{|c|c|c|c|c|c|}
\hline $\begin{array}{c}\text { Источники } \\
\text { дисперсии }\end{array}$ & $\begin{array}{c}\text { Суммы } \\
\text { квадратов }\end{array}$ & $\begin{array}{c}\text { Степени } \\
\text { свободы }\end{array}$ & $\begin{array}{c}\text { Средние } \\
\text { квадраты }\end{array}$ & $\begin{array}{c}\text { Дисперсионные } \\
\text { отношения, } \mathrm{F}\end{array}$ & $\begin{array}{c}\text { Уровни } \\
\text { значимости, } \mathrm{P}\end{array}$ \\
\hline $\mathrm{A}: \mathrm{X}_{1}$ & 0,253659 & 1 & 0,253659 & 61,89 & 0,0043 \\
\hline $\mathrm{B}: \mathrm{X}_{2}$ & 0,00405 & 1 & 0,00405 & 0,99 & 0,3934 \\
\hline $\mathrm{AA}$ & 0,108958 & 1 & 0,108958 & 26,59 & 0,0141 \\
\hline $\mathrm{AB}$ & 0,0001 & 1 & 0,0001 & 0,02 & 0,8858 \\
\hline $\mathrm{BB}$ & 0,0362111 & 1 & 0,0362111 & 8,84 & 0,0589 \\
\hline $\mathrm{R}^{2}, \%$ & \multicolumn{5}{|l}{} \\
\hline
\end{tabular}

Plot of y2

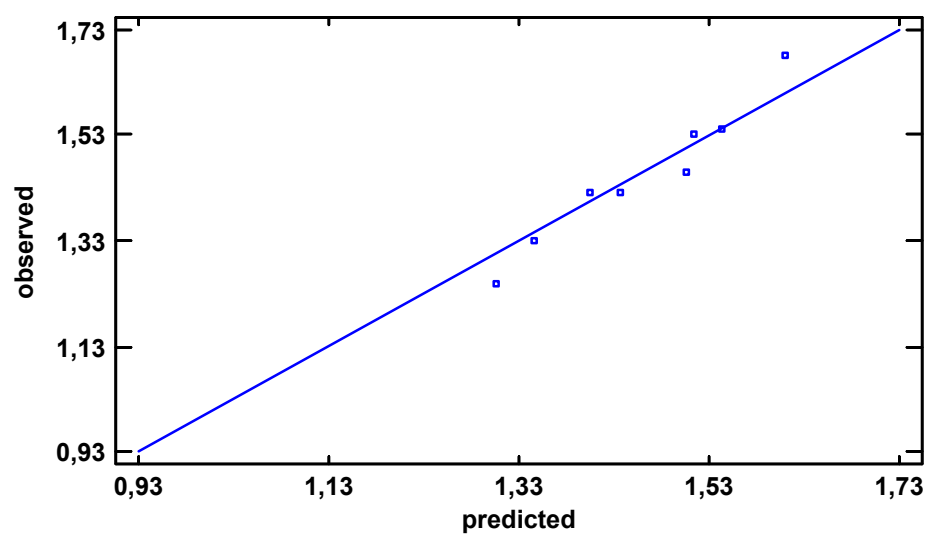

Рис. 3. Сравнение экспериментальных и расчетных значений $Y_{2}$ углеродных сорбентов из луба коры березы 


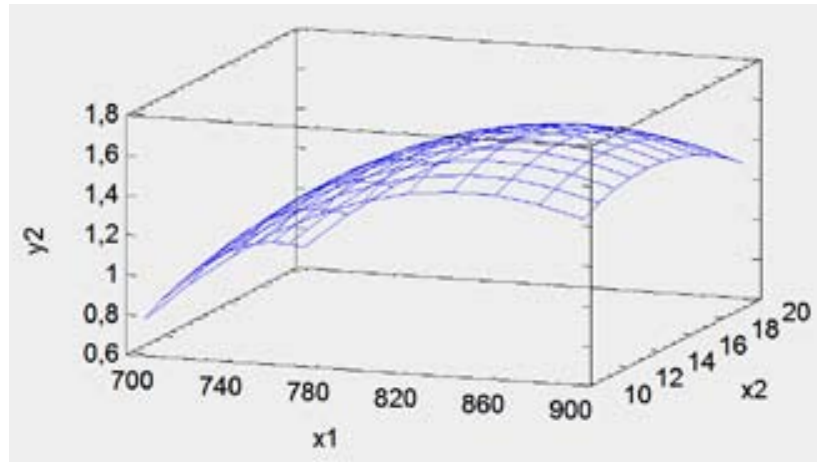

Рис. 4. Поверхность отклика зависимости объема порҮ 2 от условий процесса $\mathrm{X}_{1}$ и $\mathrm{X}_{2}$

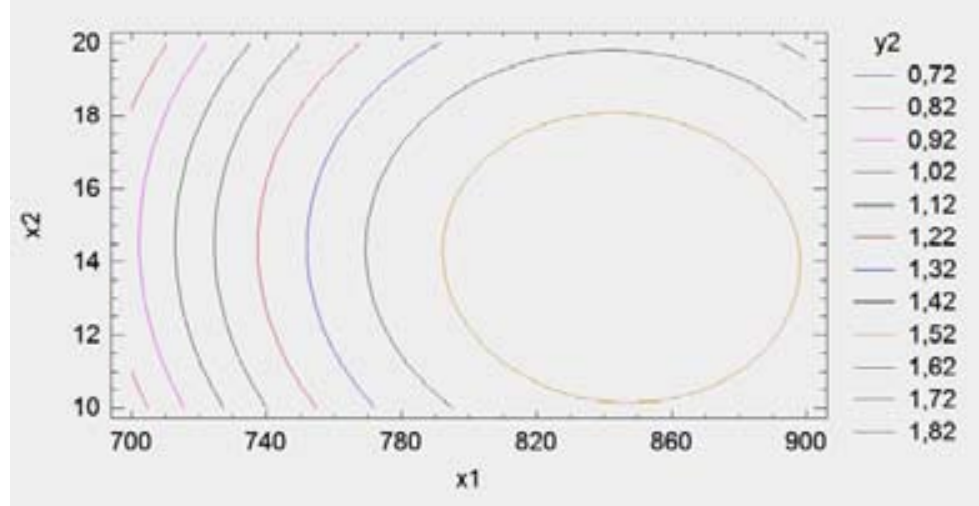

Рис. 5. Изолинии поверхности отклика зависимости объема пор $Y_{2}$ от условий процесса $X_{1}$ и $X_{2}$

$845^{\circ} \mathrm{C}$ и продолжительности активации 14 мин. Прогнозируемый объем пор при этих условиях составляет $1,62 \mathrm{~cm}^{3} / \Gamma$.

Анализ влияния условий парогазовой активащии

на адсорбционную активность по йоду углеродных сорбентов

из луба коры березы

В табл. 4 приведены результаты дисперсионного анализа для получения математических моделей (уравнений регрессии) и вычисления оптимальных условий, связывающих характер зависимостей исходных переменных $\mathrm{X}_{1}$ (температуры активации) и $\mathrm{X}_{2}$ (продолжительность активации) с выходным параметром $\mathrm{Y}_{3}$ (активности по йоду).

Уравнение регрессии, аппроксимирующее зависимость $\mathrm{Y}_{3}$ от $\mathrm{X}_{1}$ и $\mathrm{X}_{2}$, имеет следующий вид:

$$
\begin{aligned}
& Y_{3}=-1342,9+3,272 X_{1}+5,727 X_{2}-0,001932 X_{1}{ }^{2}- \\
& -0,00180 X_{1} X_{2}-0,1356 X_{2}^{2} .
\end{aligned}
$$

Выполненный дисперсионный анализ показал, что наиболее существенный вклад в суммарную дисперсию адсорбционной активности по йоду вносит фактор $\mathrm{X}_{1}$ (температура). Вклад 
влияния температуры характеризуется максимальной величиной критерия эффективности $\mathrm{F}$ $(401,86)$ и минимальным уровнем значимости $\mathrm{P}(0,0003)$. При этом коэффициент детерминации $\mathrm{R}^{2}$ составляет $98,8 \%$.

Близость «экспериментальных точек» выходного параметра адсорбционной активности по йоду к прямой линии, соответствующей расчетным (прогнозируемым) значениям адсорбционной активности по йоду, указывает на хорошие прогностические свойства модели (уравнение регрессии). Этот результат корреспондируется с величиной коэффициента детерминации $\mathrm{R}^{2}$ (квадрат выборочного коэффициента), близкого по смыслу к коэффициенту множественной корреляции между выходным параметром адсорбционной активности по йоду и переменными факторами. Высокое значение коэффициента $\mathrm{R}^{2}=98,8$ \% указывает на хорошее качество аппроксимации результатов наблюдений уравнением (3).

Прогностические свойства уравнения (3) видны из близости результатов опытов (столбец 7 в табл. 1) и прогнозируемыми моделью значениями (столбец 8). На это же указывает достаточно высокое значение коэффициента детерминации $R^{2}=98,8 \%$. Наглядное представление о качестве модели дает рис. 6. Близость точек к прямой подтверждает хорошее качество модели.

Таблица 4. Результаты дисперсионного анализ для адсорбционной активности по йоду ( $\left.\mathrm{Y}_{3}\right)$

\begin{tabular}{|c|c|c|c|c|c|}
\hline $\begin{array}{c}\text { Источники } \\
\text { дисперсии }\end{array}$ & $\begin{array}{c}\text { Суммы } \\
\text { квадратов }\end{array}$ & $\begin{array}{c}\text { Степени } \\
\text { свободы }\end{array}$ & $\begin{array}{c}\text { Средние } \\
\text { квадраты }\end{array}$ & $\begin{array}{c}\text { Дисперсионные } \\
\text { отношения, } \mathrm{F}\end{array}$ & $\begin{array}{c}\text { Уровни } \\
\text { значимости, } \mathrm{P}\end{array}$ \\
\hline $\mathrm{A}: \mathrm{X}_{1}$ & 647,929 & 1 & 647,929 & 401,86 & 0,0003 \\
\hline $\mathrm{B}: \mathrm{X}_{2}$ & 2,42 & 1 & 2,42 & 1,50 & 0,3079 \\
\hline $\mathrm{AA}$ & 347,795 & 1 & 347,795 & 215,71 & 0,0007 \\
\hline $\mathrm{AB}$ & 0,2025 & 1 & 0,2025 & 0,13 & 0,7465 \\
\hline $\mathrm{BB}$ & 18,1453 & 1 & 18,1453 & 11,25 & 0,0439 \\
\hline $\mathrm{R}^{2}, \%$ & \multicolumn{5}{|l|}{} \\
\hline
\end{tabular}

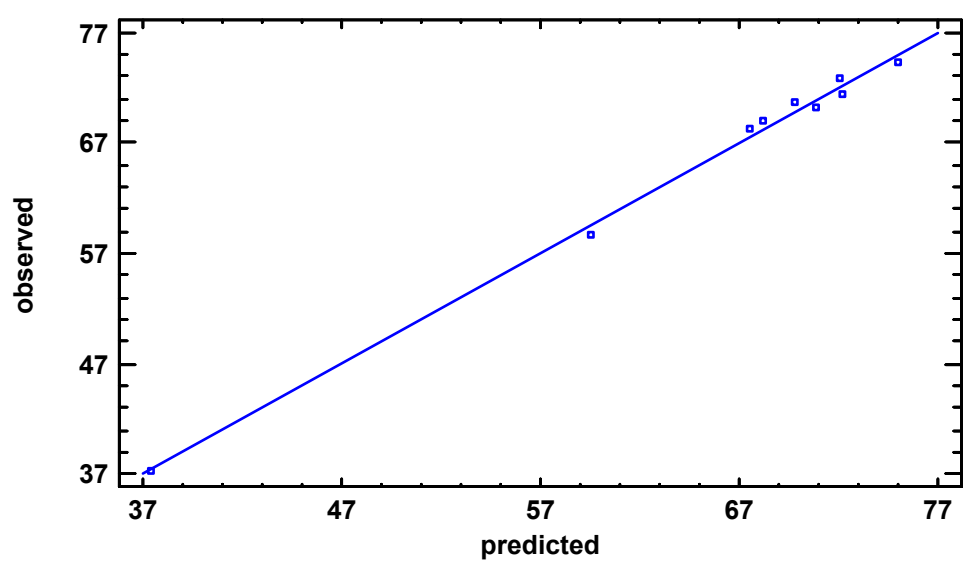

Рис. 6. Сравнение экспериментальных и расчетных значений $Y_{3}$ углеродных сорбентов из луба коры березы 
На рис. 7 приведены поверхности отклика, иллюстрирующие зависимость адсорбционной активности по йоду $\mathrm{Y}_{3}$ от переменных факторов $\mathrm{X}_{1}$ и $\mathrm{X}_{2}$. Поверхность отклика отражает факторные координаты точки, в которой значение выходного параметра адсорбционной активности по йоду достигает оптимальной величины в зависимости от влияния переменных факторов.

Из представленных на рис. 8 изолиний поверхности отклика, зависимости адсорбционной активности по йоду $\mathrm{Y}_{3}$ от условий процесса $\mathrm{X}_{1}$ и $\mathrm{X}_{2}$ видна точка, в которой $\mathrm{Y}_{3}$ достигает максимального значения, - в центре эллипса. Координаты этой точки соответствуют температуре процесса активации $839,6^{\circ} \mathrm{C}$ и продолжительности активации 15,5 мин. Прогнозируемая адсорбционная активность по йоду при этих условиях 75,2\%.

Следует отметить, что между объемом пор и адсорбционной активностью существует связь, характеризуемая коэффициентом парной линейной корреляции 0,926 . Поэтому как графики, так и результаты оптимизации этих параметров близки.

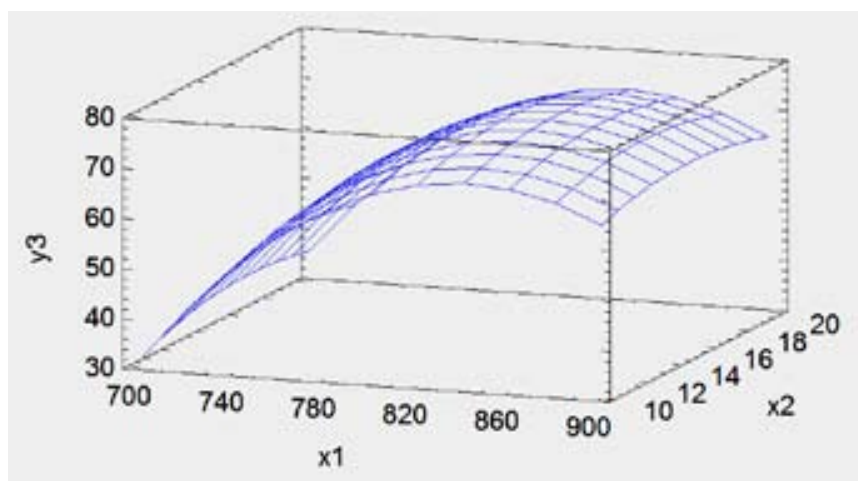

Рис. 7. Поверхность отклика зависимости адсорбционной активности по йоду $\mathrm{Y}_{3}$ от условий процесса $\mathrm{X}_{1}$ и $\mathrm{X}_{2}$

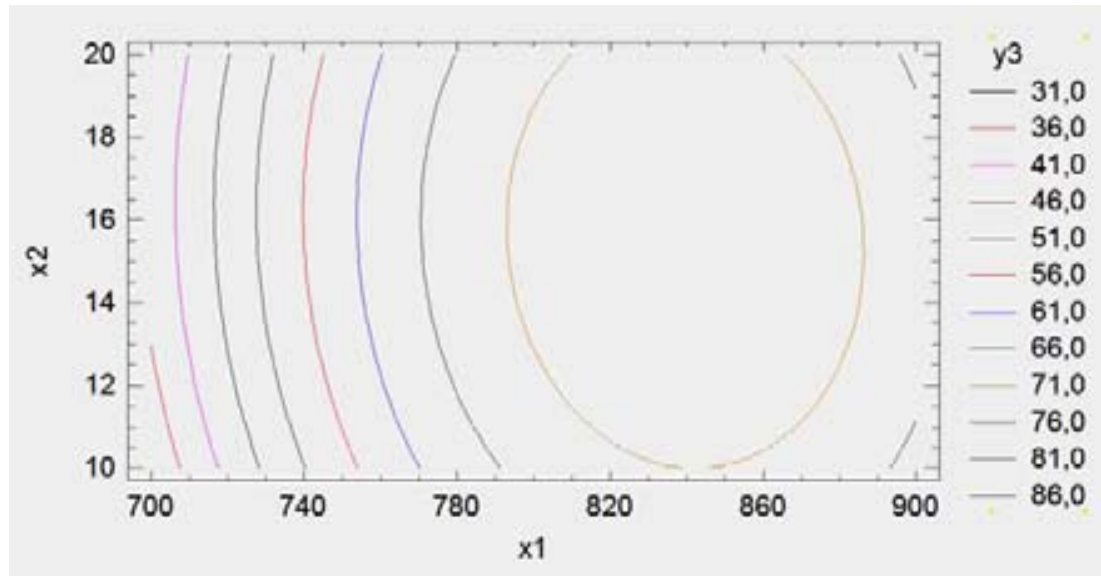

Рис. 8. Изолинии поверхности отклика, зависимости адсорбционной активности по йоду $\mathrm{Y}_{2}$ от условий процесса $\mathrm{X}_{1}$ и $\mathrm{X}_{2}$ 


\section{Заключение}

В результате проведенного экспериментально-статистического анализа получены математические модели, описывающие влияние основных исходных переменных (температуры и продолжительности процесса) на выходные параметры углеродных сорбентов, получаемых при парогазовой карбонизации-активации луба коры березы.

Показано, что оптимальное прогнозируемое значение объема пор - 1,61 cм $3 / \Gamma$ - достигается согласно произведенным вычислениям по математической модели в точке оптимума, соответствующей температуре парогазовой активации $845^{\circ} \mathrm{C}$ и продолжительности процесса 14,1 мин.

Получен прогноз оптимальных условий, при которых будут достигнуты условия формирования пористой структуры углеродных сорбентов из луба коры березы, приводящие к наибольшей активности по сорбции йода. Наилучшие результаты процесса прогнозируются при соблюдении следующих условий:

- температура активации $-839,6^{\circ} \mathrm{C}$;

- продолжительность процесса активации - 15,5 мин;

- прогнозируемая максимальная адсорбционная активность по йоду - 75,2 \%.

Установлено, что проведение процесса парогазовой карбонизации-активации луба коры березы в оптимальных условиях позволит получать углеродные сорбенты, соответствующие по своим характеристикам промышленному углеродному сорбенту БАУ, получаемому из древесины березы с объемом пор по воде 1,61 см³/Г и адсорбционной активности по йоду 75,2 \%.

\section{Список литературы}

1. FAO [Food and Agriculture Organization of the United Nations) (2011) State of the world's forests. 2011. FAO, Rome.

2. Wigginton N., Yeston J., Malakoff D. Infographic: World of waste // Science. 2012. N 337. P. 664-667.

3. Кузнецов Б. Н., Левданский В. А., Кузнецова С. А. Химические продукты из древесной коры / М-во образования и науки Рос. Федерации, Сиб. федер. ун-т. Красноярск: СФУ, 2012. $259 \mathrm{c}$.

4. Harkin J.M., Rowe J.W. Bark and its possible uses. Research note. Forest Products Laboratory. http://www.treesearch.fs.fed. us/Pubs/5760. Accessed 30 June 2012

5. Tuck C.O., Perez E., Horvath I.T., Sheldon R.A., Poliakoff M. Valorization of biomass: deriving more value from waste // Science. 2012. №337. P. 695-699.

6. Иванов И.П., Судакова И.Г., Иванченко Н.М., Кузнецов Б.Н. Изучение свойств активных углей из зерненной коры лиственницы // Химия растительного сырья. 2011. №1. C. $81-86$

7. Phussadee Patnukao1, Apipreeya Kongsuwan, Prasert Pavasant Batch studies of adsorption of copper and lead on activated carbon from Eucalyptus camaldulensisDehn. bark // Journal of Environmental Sciences. 2008. N20. P. 1028-1034.

8. Sun Y., Webley P.A. Preparation of activated carbons from corncob with large specific surface area by a variety of chemical activators and their application in gas storage // ChemEng J. 2010. N162. P. 883-892.

$$
-531-
$$


9. Yong Xiao, Hanwu Dong, Chao Long, MingtaoZheng, Bingfu Lei, Haoran Zhang, Yingliang Liu Melaleuca bark based porous carbons for hydrogen storage // International Journal of Hydrogen Energy. 2014. V.39, N22/ P. 11661-11667.

10. Пен Р.3. Планирование эксперимента в Statgraphics Centurion. Красноярск, 2014. 293 с. / Pen R.Z. Experimental Design in Statgraphics Centurion. Krasnoyarsk, 2014. 293 p.

11. ГОСТ 6217-74. Уголь активный древесный дробленый. Технические условия. М.: Издво стандартов, 1997. 9 с. 